\title{
Headache, epilepsy and photosensitivity: how are they connected?
}

\author{
Dorothée G. A. Kasteleijn-Nolst Trenité • Alberto Verrotti • \\ Alessia Di Fonzo • Laura Cantonetti • Raffaella Bruschi • \\ Francesco Chiarelli $\cdot$ Maria Pia Villa $\cdot$ Pasquale Parisi
}

Received: 9 April 2010/Accepted: 25 May 2010/Published online: 21 October 2010

(C) Springer-Verlag 2010

\begin{abstract}
Although headache and epilepsy have often been associated, the precise electroclinical and pathophysiological interaction between these disorders and in particular its relations with photosensitivity is as yet to be fully understood in adults or children. The association between headache and epilepsy commonly occurs in all types of epilepsy and not only in occipital epilepsy. Generally, peri-ictal headache is often neglected, regardless of its severity, because patients are more concerned about their seizures. Altered cerebral cortex excitability may be the link between these two conditions and photosensitivity shows this. The physician should bear this association in mind when dealing with epileptic and migraine patients so as to be able to offer such patients an accurate diagnosis and appropriate treatment; this should be borne in mind when declaring epileptic patients 'seizure free'. To date
\end{abstract}

D. G. A. Kasteleijn-Nolst Trenité

Department of Medical Genetics, Wilhelmina Children's

Hospital, Utrecht University Medical Centre,

Utrecht, The Netherlands

R. Bruschi · M. P. Villa $\cdot$ P. Parisi $(\bowtie)$

Chair of Paediatrics, Child Neurology, Headache Paediatric

Center, Paediatric Sleep Disorders, II Faculty of Medicine,

"Sapienza University", c/o Sant'Andrea Hospital,

Via di Grottarossa 1035-1039, Rome, Italy

e-mail: pasquale.parisi@uniroma1.it; parpas@iol.it

A. Verrotti - A. Di Fonzo · F. Chiarelli

Chair of Paediatrics, Paediatric Department,

University of Chieti, Chieti, Italy

L. Cantonetti

Unit of Paediatric Rehabilitation, Department of Neuroscience,

Bambino Gesù Children's Hospital, IRCCS,

Santa Marinella, Rome, Italy neither the International Headache Society nor the International League against Epilepsy mention that headache/ migraine may, on occasion, be the sole ictal epileptic manifestation. Lastly, studies designed to investigate the triggering role of photosensitivity in both headache and epilepsy are warranted.

Keywords Peri-ictal headache $\cdot$ Ictal epileptic headache · PPR - Photosensitivity - Headache - Migraine - Epilepsy · Migralepsy $\cdot$ Hemicrania epileptica

\section{Introduction}

Although we do not yet fully understand the underlying mechanisms shared by headache, epilepsy and photosensitivity, all three conditions are related to intermittent changes in brain excitability and are known to facilitate each other. This interaction emerges, for example, from EEG abnormalities and related clinical phenomena evoked by intermittent photic stimulation (IPS), which can induce photoparoxysmal EEG responses (PPR), migraine and epileptic seizures.

Since epileptic patients are usually more concerned about their seizures than about headache, headache symptoms are often neglected by both patients and treating physicians. This might explain why these disorders are generally viewed as two separate paroxysmal diseases that are considered to express, at most, epidemiological and clinical comorbidity [1-12].

Only in idiopathic occipital lobe epilepsies, a subgroup of epilepsies, is the close association between migraine and epilepsy acknowledged [13-18]: both occipital epilepsies and migraine are characterized by visual aura followed by headache and other autonomic symptoms. Recognition of 
headache as an epileptic manifestation per se represents a challenge yet [19-21].

The aim of this article was therefore to review the literature on the clinical and EEG characteristics of peri-ictal headache in children with epilepsy, and to discuss the temporal and etiopathogenetic relationships between headache, epilepsy and specifically photosensitivity.

\section{Definitions}

In this review, we have used the following definitions:

- Headache is pain located above the orbitomeatal line.

- Migraine is a recurrent headache disorder that manifests itself in attacks lasting 4-72 h; typical characteristics of migraine are unilateral location, pulsating quality, moderate or severe intensity, aggravation by routine activity and association with nausea and/or photophobia and phonophobia. In children, migraine attacks may last 1-72 h [22].

- Epilepsy is a chronic neurological condition characterized by recurrent manifestations of excessive and/or hypersynchronous, usually self-limited activity of neurons in the brain [23].

- Photosensitivity is an epileptic reaction to visual stimuli. A distinction can be made between the epileptiform EEG responses to IPS during an EEG recording (the so-called photoparoxysmal responses: PPRs) and clinical signs and symptoms, either evoked by IPS or by visual stimuli in daily life, such as disco lights, TV and video games. PPRs are abnormal, highly heritable, electroencephalographic responses of the cortical human brain to specific flash frequencies or other visual stimuli. If a PPR is found, the patient is considered IPS-sensitive or photosensitive. Visual sensitivity is defined as the susceptibility to seizures, which are triggered by the physical characteristics of visual stimuli and not by their perceptual properties, i.e., reflex seizures induced by the cognitive effects of visual stimuli [24].

- The term migralepsy was introduced by Lennox and Lennox in 1960 (although they attributed it to Dr Douglas Davidson) to describe a condition wherein "ophthalmic migraine is followed by symptoms characteristic of epilepsy" [25]. The term itself comes from the combination of the terms migraine and epilepsy. Since then, approximately 50 cases of migralepsy have been described, the majority of which have been the object of debate. Panayiotopoulos [26], in particular, argued that although "there should be no reason why epileptic seizures, so vulnerable to extrinsic and intrinsic precipitating factors, could not be susceptible to cortical changes induced by migraine and vice versa". Most of the reported cases of migralepsy are complicated and do not have an unequivocal migraineepilepsy sequence; they may thus be considered as genuine occipital seizures that resemble migraine with aura and are characterized by visual aura, headache and other autonomic symptoms [27]. Headache itself may even be the epileptic "aura" of a seizure, as has been shown in the case description of a patient with partial status epilepticus in occipital lobe epilepsy [19, 27].

- Migralepsy has been included in the ICHD-2 and ILAE classifications. Despite the skepticism surrounding the concept of migralepsy, in which two separate disorders are presumed to occur in succession, migraine-triggered seizures have been included in the recent ICHD-II (1.5.5) as a complication of migraine based on the fulfillment of two criteria [22]: (a) for migraine with aura; (b) for the occurrence of an epileptic attack during or within $1 \mathrm{~h}$ of a migraine aura. However, migralepsy is not included in the currently used International League Against Epilepsy (ILAE) seizure classification dating from 1981, or in recent recommendations of the ILAE Commission on Classification and Terminology (2009) [21, 28-30].

- Seizure-related headache (SRH) may be pre-, post- or peri-ictal, i.e., they may precede, follow or occur in temporal proximity of the actual seizure manifestations.

- Hemicrania epileptica is recognized by ICHD-2 classification (coded as 7.6.1) as an "ictal headache" (ipsilateral to the ictal EEG discharge) "synchronously" with a seizure (partial epileptic seizure) recognized by ILAE classification.

- The term "ictal epileptic headache" [19, 21, 28-30] recognizes headache as "the sole epileptic phenomenon" without any other associated ictal epileptic signs/ symptoms recognized by ILAE.

\section{Comorbidity of migraine and epilepsy}

Epidemiology of comorbidity

Individuals with epilepsy are 2.4 times more likely to suffer from migraine than their relatives without epilepsy [3]. Baulac [5] found an epilepsy prevalence of 5.9\% in a population of 1830 migraine patients. In another study, the prevalence of epilepsy in people with migraine ranged from 1 to $17 \%$, with a median of $5.9 \%$, which is substantially higher than the $0.5 \%$ prevalence of epilepsy in the general population [6, 12].

Marks and Ehrenberg [7] found that 79 out of 395 (20\%) patients with epilepsy also had migraine, according to the 
first International Headache Classification (IHS). In the vast majority of patients with both disorders (84\%), the attacks occurred independently. However, they did recognize that migraine aura symptoms very often resemble symptoms of an epileptic seizure.

Very recently, Toldo et al. [1] demonstrated again the strong association between migraine and epilepsy: indeed, they found that the risk of epilepsy in migraineurs was 3.2 times higher than in subjects with tension-type headache, with no significant difference between migraine with and without aura. Focal epilepsies prevailed in cases characterized by comorbidity $(76.8 \%)$. Migraineurs affected by focal epilepsies $(36 / 56)$ were three times more likely to develop cryptogenic epilepsy (75\%) than idiopathic epilepsy $(25 \%)$.

Peri-ictal headache in epilepsy

According to their temporal relationship to the epileptic attack, peri-ictal headaches can be divided into pre-ictal- or post-ictal. Inter-ictal headache occurs by definition between two epileptic seizures. The boundaries between these three subtypes are often not well defined and also in clinical practice difficult to discriminate. In 100 consecutive patients with pharmacologically refractory partial epilepsy, 11 reported pre-ictal headache of which 7 had early pre-ictal headache (up to $30 \mathrm{~min}$ before seizure onset) and 4 had prodromic pre-ictal headache $(24 \mathrm{~h}$ to $30 \mathrm{~min}$ before seizure onset). Pre-ictal headache was ipsilateral to the seizure focus in all but one case and had migrainous features in four cases [31]. Pre-ictal headache has also been reported in previous studies [31-35] as having a lateralizing value.

In children, the prevalence of headache was higher than in adults: in 101 children (aged 5-18 years) with generalized tonic-clonic or partial seizures peri-ictal headaches were reported by $41 \%$ (29\%, post-ictal only; $5 \%$, pre-ictal only; $7 \%$, both). Clear migrainous features were present in $50 \%$ of pre-ictal and $58 \%$ of post-ictal headaches. Most children described bilateral headaches [36].

Post-ictal headache may be of the migraine type, tension type or unclassified type of headache [32, 37, 38]. Several researchers have pointed out that post-ictal headache symptoms resemble those of migraine; they include pounding pain and accompanying nausea, photophobia and phonophobia in a significant proportion of patients $(41-56 \%)$ [10, 35, 39, 40]. Previous reports have suggested that migraine-type headaches often occur during post-ictal periods in epilepsy syndromes, such as occipital lobe epilepsy [41-43]. In a recent study, Ito [44] found that the incidence of post-ictal headache was significantly higher in patients with generalized tonic-clonic seizures than in those with other types of seizures, thus confirming the findings of a number of previous studies [10, 37, 39, 40, 45, 46]. Generalized tonic-clonic seizures were more frequently followed by other post-ictal headaches than by migrainelike post-ictal headache, a finding that is in keeping with other reports [33, 39]. It is possible that the generalization of the seizure discharge may induce other pathophysiological changes in the whole brain structure. In another study [9] conducted on a cohort of 227 adult patients with a mean seizure frequency of 2.46 per month, 50 patients (22\%) said that they had at least one headache episode, 45 (19.8\%) had an inter-ictal headache, $11(4.8 \%)$ a peri-ictal headache, while $5(2.2 \%)$ had both an inter-ictal and peri-ictal headache. Neither the prolonged course nor the severity of epilepsy is likely to have an impact on post-ictal headache.

Post-ictal headache may also occur after complex partial seizures originating in the temporal lobe [35, 47]. In the study by Ito [44], the occurrence of migraine-like post-ictal headache was significantly related to the region of the epileptogenic focus: occipital lobe epilepsy was strongly correlated with migraine-like post-ictal headache. The occipital lobe is deemed to be the brain structure most responsible for the development of migraine [47, 48]. Although post-ictal headache was less frequent in patients with temporal lobe epilepsy, migraine-like post-ictal headache occurred more frequently than other post-ictal headaches. The posterior temporal cortex locate close to the occipital cortex and the anatomic connections between the mesial temporal structures and occipital lobe are abundant [49, 50]. When both the temporal lobes and occipital lobes are firing, the structures related to migraine symptoms may rapidly become involved [47-50].

Regarding "ictal epileptic headache", alone or associated with other ictal epileptic manifestations, it should be borne in mind that headache may be associated with ictalsensory and motor features more frequently than the literature suggests; indeed, this association might be strongly underestimated owing to impaired consciousness during complex partial seizures with or without secondary generalization. In this respect, an ictal EEG (during the migraine attack) should be recommended in every migraine patient, even in subjects who are not known to be epileptic. During an inter-ictal EEG (or video-EEG) recording, particular attention should always be paid to the ictal onset of the clinical EEG findings (and not the EEG abnormalities alone, which are relatively common) during IPS, performed according to the standardized international protocol [28].

\section{Role of photosensitivity}

Although occipital lobe epilepsy already has much in common with migraine (visual aura, positive and negative ictal signs and autonomic disturbances such as pallor and 
vomiting), the photosensitive variant of occipital epilepsy and photosensitive epilepsy in general have even more similarities: in addition to the above, a high prevalence in women (female:male 3:2) and a sensitivity to flickering light stimuli and striped patterns with provocation of attacks is found.

Photosensitive epilepsy patients have seizures provoked by visual stimuli such as flickering sunlight, disco lights, TV, etc. [51]. During IPS in the EEG laboratory, an epileptiform EEG reaction or so-called PPR can be elicited in these patients with the highest prevalence in adolescents.

In EEG studies in 137 children and adolescents affected by primary headache [52], specific EEG abnormalities were reported in $11.7 \%$ of the subjects, with a significantly heterogeneous distribution across the groups. Children affected by migraine with aura, in particular, exhibited a higher occurrence of specific inter-ictal abnormalities (43.5\%); a PPR was detected in over one-third of the migraine with aura subgroup, which is a far higher figure than that reported in a healthy population of children (1.4\%) and in epilepsy patients in general (5\%) [53]. In another study that investigated the PPR frequency and type in 263 primary headache-affected children and adolescents between 7 and 18 years of age, Wendorff et al. [54] found that the PPR frequency did not differ significantly in the three main types of headaches, ranging from $6.7 \%$ (tension-type headache) to $8.9 \%$ (migraine without aura). However, PPR proved to be most frequent $(17.6 \%)$ in the migraine with aura group of patients under 12 years of age, and in 7 out of 17 patients with both migraine and PPR, photogenic stimuli were the most frequent triggering factors of the migraine attack, compared with 10 out of 100 controls $(p<0.01)$.

As regards the possible role of photosensitivity in both headache and epilepsy, a striking example is our reported case of a 14-year-old photosensitive girl. Her first generalized tonic-clonic seizure was preceded and followed by a 3-day epilepticus status migrainosus, which resolved only after intravenous administration of diazepam; continuous EEG recording during the diazepam infusion showed suppression of the epileptiform discharges over the right occipital region, while the headache resolved completely [19]. The "migraine" status in that girl had been caused by prolonged television viewing (a new, large-screen television) and visiting an exhibition that contained a lot of strong visual stimuli, such as huge boards with dark black text on a super white background and a variety of primary strong colors in various pictures by artists. Recent publications described "pediatric confusional migraine" cases in which valproate was efficacious. However, they did not investigate whether there was also photosensitivity $[55,56]$.
The likelihood of an ictal (epileptic) headache appears to be greater when both migraine and epilepsy occur in the same family [27, 30]. Photic stimulation during EEG recording in patients with a history of headache and with a family history of migraine and epilepsy may contribute to a correct diagnosis and optimal care of the patient [30]. It can be very difficult to discriminate between photosensitive epilepsy and migraine with clinical history alone, because sensitivity to light (photophobia) and provocation by strong lights are difficult to disentangle for the patient as well as the treating physician.

Propagation of PPR is associated with increased excitability of the occipital cortex [57]. In this regard, it should be borne in mind that the accumulated burden of migraine seems to cause slight alterations in the physiology of the visual cortex and an increase in alpha rhythm variability up to $72 \mathrm{~h}$ before the next migraine attack [58].

Phosphenes may occur following electrical stimulation of the retina, optic nerve or visual cortex, as well as during and after IPS. Moreover, IPS induces "flashes and phosphenes" as well as migraine and seizures. Flashes, phosphenes and other positive or negative visual manifestations are often part of the clinical picture in migraine and occipital epilepsy. Neurons of the retina and cortex are sensitive to ionizing agents, to which they respond with transient functional changes. In this regard, the phosphenes described by astronauts during space missions are comparable to those experienced by the totally blind and by sighted subjects with migraine or epileptic seizures that originate in the visual cortex [59].

Lastly, the perception of migraine headache pain is exacerbated by exposure to ambient light, compared with the pain level in the dark, and hence photophobia occurs. Photophobia is one of the most well-known features of migraine ( $>80 \%$ of chronic migraineurs [60] and also very often found in epilepsy-related headaches: in $>50 \%$ of cases it occurs in pre-ictal, post-ictal or even inter-ictal headache [61]. The authors of one study recently postulated that exacerbation of migraine headache by light is driven by photic signals transmitted from the retina via the optic nerve to central neurons that process nociceptive signals from the meninges [62]. Those authors found that exacerbation of headache by light was preserved in blind migraineurs who rely primarily on non-image-forming photoreception, but was absent in those who lost their optic nerve or eyes. They also found that light modulated the activity of a subset of trigeminovascular thalamic neurons that receive input from the retina and project to multiple cortical areas [62]. The idea that intrinsically photosensitive retinal ganglion cells are involved in migraine photophobia is supported by the fact that light-induced exacerbation of migraine headache was preserved in blind 
individuals who could sense light in the face of severe degeneration of rod and cone photoreceptors [62].

\section{Underlying mechanisms for the overlap between headache, epilepsy and photosensitivity}

From both a genetic [63-65] and clinical [19, 20, 30] point of view, the concept of a "continuum", or maybe even a complete "overlap", between headache and epilepsy is gaining increasingly strong evidence-based support. Indeed, a novel SCN1A L263V mutation in a family with co-segregating hemiplegic migraine (FHM) and epilepsy [65] and a $\mathrm{Na}^{+}, \mathrm{K}^{+}$-ATPase pump defect [66] have been documented. Moreover, up to $75 \%$ of FHM families have a mutation in the P/Q-type calcium channel $\mathrm{Ca}(\mathrm{v}) 2.1$ subunit CACNA1A gene on chromosome 19p13, while a candidate region of $9.95 \mathrm{cM}(5.96 \mathrm{Mb})$ between markers GATA152H04 and D9S253 located at chromosome 9q21q22 was identified in a large Belgian family with occipitotemporal lobe epilepsy associated with migraine with visual aura [64].

A migraine/headache attack can originate at either the cortical or subcortical level, whereas an epileptic focus arises cortically and can only be modulated at the subcortical level [28, 29, 67]. Two mechanisms have been hypothesized to explain headache/migraine as a sole "ictal epileptic manifestation" [21, 28, 68]: (1) a subclinical epileptic discharge might activate the trigeminovascular system, resulting in a migraine/headache without any other associated cortical epileptic sign or symptom; (2) central autonomic networks (whether cortical or subcortical) have a lower threshold for epileptogenic activation than those that produce a focal cortical semiology. For example, in the Panayiotopoulos syndrome, ictal epileptic autonomic symptomatology appears to pertain to any epileptogenic cortical onset zone, be it occipital, frontotemporal or frontal [69]. Therefore, seizures might remain purely autonomic if ictal neuronal activation of non-autonomic cortical areas fails to reach the symptomatogenic threshold. This possible mechanism, first suggested for the Panayiotopoulos syndrome, might represent another mechanism that leads to headache/migraine as a sole "ictal epileptic manifestation" $[21,28]$.

It should be stressed that, as occurs for autonomic manifestations (vomiting, tachycardia, etc.), children are far more likely to develop migraine as the sole epileptic manifestation than adults. A clinical observation (not published) is also that during extensive photic stimulation, especially children with generalized PPRs complain of headache. Why the occipital lobe stimulates autonomic symptoms and signs to a much greater extent than other cortical areas is still unknown. Although a correlation between the microstructure and function of an area and its connections has been established for many cortical regions, particularly for primary sensory and motor areas [70], very little is as yet known about the anatomofunctional pathways linking the cerebral cortex and cortico-subcortical vegetative/autonomic neural networks.

In the last decade, the roles of cortical spreading depression (CSD) and trigeminovascular system (TVS) activation in the physiopathology of migraine have been clearly documented [71]. The "sine qua non" condition for headache (pain phase) is TVS activation, which is believed to be the only neural pathway that induces migraine (with or without aura), while CSD (or, more rarely, subcortical spreading depression) is responsible for TVS activation [72]. Epileptic discharges and CSD stimulate each other in experimental conditions [73]. The threshold required for the onset of CSD has recently been suggested $[21,28,29,68]$ to be lower than that required for an epileptic seizure. In other words, the onset of epileptic seizure may facilitate the onset of CSD to a greater degree than the onset of CSD facilitates the onset of epileptic seizure. This may explain why, in the clinical context, we are more likely to observe epileptic patients with "comorbid" (peri-ictal and inter-ictal) migraine than migraine subjects with "comorbid" epilepsy [28].

Another important evidence of overlap between epilepsy and migraine is the current FDA approved use of AEDs such as valproate (VPA) and topiramate (TPM) in migraine patients [74].

TPM and levetiracetam (LEV) were recently found to be able to normalize migraine-type impaired cortical excitability [75]. Vice versa, drugs developed for migraine prevention, such as the b-blocker propranolol, the $\mathrm{Ca}^{2+}$ channel blocker flunarizine and the tricyclic antidepressant amitriptyline, do show some anti-epileptic effect [75].

Maybe, as in epilepsy, the imbalance between excitatory and inhibitory transmission is selectively expressed in brain regions also involved in the pathophysiology of migraine, such as specific brainstem structures and cortical areas. Although it is well known that AEDs exert their anticonvulsant action by targeting these pathogenetic mechanisms, the reasons why not all AEDs are equally effective in the treatment of migraine are still unknown.

Another interesting finding is that valproate (VPA), one of the most efficacious drugs in migraine prophylaxis, is also the drug of first choice in epilepsy patients with photosensitivity [76]. We have seen several photosensitive patients with IGE, who showed headache complaints related to visual stimuli again, after having withdrawn the VPA medication (see also reference [30]).

In summary, a subclinical spontaneous (epileptic foci or CSD) $[21,28,29]$ or induced (IPS or other visual stimulations) [30, 76] cortical event might, depending on the cortical or subcortical neural network threshold and on the 
speed and direction of propagation, result in a seizure, in migraine or in both. In exceptional cases, which occur almost exclusively in childhood, the sub-clinical focus might consist of an epileptic focus that activates the TVS, thereby inducing migraine without any other known cortical epileptic signs or symptoms [21, 28, 29, 68, 76].

\section{Conclusion}

The comorbidity of migraine and epilepsy is well known. Epilepsy prevalence in a population with migraine is higher than prevalence of epilepsy in the general population. Ictal headache as the only manifestation of epileptic seizures is very rare, and seizures associated with "migraine-like" manifestations probably represent an epileptic event rather than episodes with both migraine and epileptic mechanisms. In all events with features of both types (migraine and epilepsy), ictal EEG recordings should be performed to document and demonstrate the real pathogenic mechanisms of these episodes. The association between headache and epilepsy commonly occurs in all types of epilepsy and not in occipital epilepsy alone. Generally, peri-ictal headache is often neglected, regardless of its severity, because patients are more concerned about their seizures. Altered cerebral cortex excitability may be the link between these two conditions and photosensitivity shows this. The physician should bear this association in mind when dealing with epileptic and migraine patients so as to be able to offer such patients an accurate diagnosis and appropriate treatment. To date, neither the International Headache Society nor the International League against Epilepsy mention that headache/migraine may, on occasion, be the sole ictal epileptic manifestation. This is also reflected in the ICHD-II criteria for the "hemicrania epileptica" classification (coded as 7.6.1), where ... "the patient is having a partial epileptic seizure".

Based on the current knowledge and clinical experience, migralepsy (coded in ICHD-II as 1.5.5 "migraine-triggered seizure"), is highly unlikely to exist as such, but theoretically might. We therefore propose to delete this term until clear evidence is provided of its existence.

Lastly, studies designed to investigate the triggering role of photosensitivity in both headache and epilepsy are warranted.

\section{Conflict of interest None.}

\section{References}

1. Toldo I, Perissinotto E, Menegazzo F, Boniver C, Sartori S, Salviati L, Clementi L, Montagna P, Battistella PA (2010) Comorbidity between headache and epilepsy in a pediatric headache center. J Headache Pain 11(3):235-240
2. Fernández-de-Las-Peñas C, Hernández-Barrera V, CarrascoGarrido P, Alonso-Blanco C, Palacios-Ceña D, Jiménez-Sánchez S, Jiménez-García R (2010) Population-based study of migraine in Spanish adults: relation to socio-demographic factors, lifestyle and co-morbidity with other conditions. J Headache Pain 11(2):97-104

3. Lipton RB, Ottman R, Ehrenberg BL, Hauser WA (1994) Comorbidity of migraine: the connection between migraine and epilepsy. Neurology 44:28-32

4. Ottman R, Lipton RB (1994) Comorbidity of migraine and epilepsy. Neurology 44:2105-2110

5. Baulac S, Huberfeld G, Gourfinkel-An I (2001) First genetic evidence of $\operatorname{GABA}(\mathrm{A})$ receptor dysfunction in epilepsy: a mutation in the gamma2-subunit gene. Nat Genet 28:46-48

6. Andermann E, Andermann F (1987) Migraine-epilepsy relationships: epidemiological and genetic aspects. In: Andermann FA, Lugaresi E (eds) Migraine and epilepsy. Butterworths, Boston, pp 281-291

7. Marks DA, Ehrenberg BL (1993) Migraine-related seizures in adults with epilepsy, with EEG correlation. Neurology 43:2476-2483

8. Velioglu SK, Ozmenoglu M (1999) Migraine-related seizures in an epileptic population. Cephalalgia 19:797-801

9. Kwan P, Man CBL, Leung H, Yu E, Wong KS (2008) Headache in patients with epilepsy: a prospective incidence study. Epilepsia 49:1099-1102

10. Schon F, Blau JN (1987) Post-epileptic headache and migraine. J Neurol Neurosurg Psychiatry 20:1148-1152

11. Yankovsky AE, Andermann F, Mercho S, Dubeau F, Bernasconi A (2005) Preictal headache in partial epilepsy. Neurology 65:1979-1981

12. Parain D, Guerrini R, Hesdorferr D, Ryvlin P (eds) (2009) Epilepsy and migraine. Current problem in epilepsy. John Libbey Eurotext, Paris

13. Guerrini R, Genton P (2004) Epileptic syndromes and visually induced seizures. Epilepsia 45(Suppl 1):14-18

14. Ferrie CD, Beaumanoir A, Guerrini R et al (1997) Early-onset benign occipital seizure susceptibility syndrome. Epilepsia 38(3):285-293

15. Panayiotopoulos CP (1999) Visual phenomena and headache in occipital epilepsy: a review, a systematic study and differentiation from migraine. Epileptic Disord 1(4):205-216

16. Caraballo R, Cersósimo R, Fejerman N (2008) Childhood occipital epilepsy of Gastaut; study of 33 patients. Epilepsia 49(2):288-297

17. Fejerman N, Caraballo R (2008) Benign focal epilepsies in infancy, childhood and adolescence. John Libbey Eurotext, Paris

18. Caraballo R, Koutroumanidis M, Panayiotopoulos CP, Fejerman N (2009) Idiopathic childhood occipital epilepsy of Gastaut: a review and differentiation from migraine and other epilepsies. J Child Neurol 24(12):1536-1542

19. Parisi P, Kasteleijn-Nolst Trenité DG, Piccioli M, Pelliccia A, Luchetti A, Buttinelli C, Villa MP (2007) A case with atypical childhood occipital epilepsy "Gastaut type": an ictal migraine manifestation with a good response to intravenous diazepam. Epilepsia 48(11):2181-2186

20. Ghofrani M, Mahvelati F, Tonekaboni H (2007) Headache as a sole manifestation in nonconvulsive status epilepticus. J Child Neurol 22(5):660-662

21. Parisi P, Kasteleijn-Nolst Trenité DG (2010) Commentary on "migralepsy": a call for revision of the definition. Epilepsia 51(5):932-933

22. Headache Classification Subcommittee of the International Headache Society (2004) The International Classification of Headache Disorders, 2nd edn. Cephalalgia 24(Suppl):1-160

23. Engel J Jr, International League Against Epilepsy (ILAE) (2001) A proposed diagnostic scheme for people with epileptic seizures 
and with epilepsy: report of the ILAE Task Force on Classification and Terminology. Epilepsia 42:796-803

24. Kasteleijn-Nolst Trenite DG (1989) Photosensitivity in epilepsy Electrophysiological and clinical correlates. Acta Neurol Scand Suppl 125:3-149

25. Lennox WG, Lennox MA (1960) Epilepsy and related disorders. Little, Brown and Company, Boston, pp 582-587

26. Panayiotopoulos CP (1999) Differentiating occipital epilepsies from migraine with aura, acephalgic migraine and basilar migraine. In: Panayiotopoulos CP (ed) Benign childhood partial seizures and related epileptic syndromes. John Libbey \& Company Ltd, London, pp 281-302

27. Walker MC, Smith SJM, Sisodya SM, Shorvon SD (1995) Case of simple partial status epilepticus in occipital lobe epilepsy misdiagnosed as migraine: clinical, electrophysiological, and magnetic resonance imaging characteristics. Epilepsia 36:12331236

28. Parisi P (2009) Why is migraine rarely, and not usually, the sole ictal epileptic manifestation? Seizure 18:309-312

29. Parisi P (2009) Who's still afraid of the link between headache and epilepsy? Some reactions to and reflections on the article by Marte Helene Bjørk and co-workers. J Headache Pain 10:327-329

30. Piccioli M, Parisi P, Tisei P, Villa MP, Buttinelli C, KasteleijnNolst Trenité DG (2009) Ictal headache and visual sensitivity. Cephalalgia 29(2):194-203

31. Yankovsky AE, Andermann F, Mercho S1 et al (2005) Preictal headache in partial epilepsy. Neurology 65:1979-1981

32. Schon F, Blau JN (1987) Post-epileptic headache and migraine. J Neurol Neurosurg Psychiatry 50:1148-1152

33. Karaali-Savrun F, Goksan B, Yeni SN et al (2002) Seizurerelated headache in patients with epilepsy. Seizure 11:67-69

34. Forderreuther S, Henkel A, Noachtar S et al (2002) Headache associated with epileptic seizures: epidemiology and clinical characteristics. Headache 4:649-655

35. Bernasconi A, Andermann F, Bernasconi N et al (2001) Lateralizing value of peri-ictal headache: a study of 100 patients with partial epilepsy. Neurology 56:130-132

36. Cai S, Hamiwka LD, Wirrell EC (2008) Peri-ictal headache in children: prevalence and characteristic. Pediatr Neurol 39:91-96

37. Karaali-Savrun F, Goksan B, Yeni SNl et al (2002) Seizure related headache in patients with epilepsy. Seizure 11:67-69

38. D'Alessandro R, Sacquegna T, Pazzaglia P, Lugaresi E (1988) Post-epileptic headache and migraine. J Neurol Neurosurg Psychiatry 51:596-597

39. Leniger T, Isbruch K, Driesch S, Diener HC, Hufnagel A (2001) Seizure-associated headache in epilepsy. Epilepsia 42:1176-1179

40. Ito M, Schachter SC (1996) Frequency and characteristics of interictal headaches in patients with epilepsy. J Epilepsy 9:83-86

41. Ogunyemi A, Adams D (1998) Migraine-like symptoms triggered by occipital lobe seizures: response to sumatriptan. Can J Neurol Sci 25:151-153

42. Andermann F, Zifkin B (1998) The benign occipital epilepsies of childhood: an overview of the idiopathic syndromes and of the relationship to migraine. Epilepsia 39:9-23

43. Panayiotopoulos CP (1999) Elementary visual hallucinations, blindness and headache in idiopathic occipital epilepsy: differentiation from migraine. J Neurol Neurosurg Psychiatry 66:536-540

44. Ito M, Adach N, Nakamura F, Koyama T, Okamura T, Kato M, Kanemoto K, Nakano T, Matsuura M, Hara S (2004) Characteristics of postictal headache in patients with partial epilepsy. Cephalalgia 24:23-28

45. Ito $M$, Nakamura $H$, Honma $H$, Takeda $Y$, Kobayashi $R$, Miyamoto $\mathrm{Tl}$ et al (2000) Clinical factors associated with postictal headache in patients with epilepsy. Acta Neurol Scand 102:129-131

46. Schachter SC, Richman K, Loder E, Belk S (1995) Self-reported characteristics of postictal headaches. J Epilepsy 8:41-43

47. Lauritzen M (1994) Pathophysiology of the migraine aura. The spreading depression theory. Brain 117:199-210

48. Panayiotopoulos CP (1987) Difficulties in differentiating migraine and epilepsy based on clinical EEG findings. In: Andermann F, Lugaresi E (eds) Migraine and epilepsy. Butterworths, Boston, pp 31-46

49. Blume WT, Wiebe S (2000) Occipital lobe epilepsies. In: Williamson PD, Siegel AM, Roberts DW, Thadani VM, Gazzaniga MS (eds) Neocortical epilepsies. Advances in neurology. Lippincott/Williams \& Wilkins, Philadelphia, pp 173-187

50. Pandya DN, Yeterian EH (1985) Architecture and connections of cortical association areas. In: Peters A, Jones EG (eds) Cerebral cortex association and auditory cortices. Plenum, New York, pp 3-62

51. Kasteleijn-Nolst Trenite DG, Binnie CD, Harding GF, Wilkins A (1999) Photic stimulation: standardization of screening methods. Epilepsia 40(Suppl 4):75-79

52. Piccinelli P, Borgatti R, Nicoli F et al (2006) Relationship between migraine and epilepsy in paediatric age. Headache 46:413-421

53. Kasteleijn-Nolst Trenité DGA, Silva LCB, Manreza MLG (2003) Prevalence of photo paroxysmal EEG responses in normal children and adolescents in Teofile Otoni, Brazil: 2001-2002. Epilepsia 44(Suppl 8):48

54. Wendorff J, Juchniewicz B (2005) Photosensitivity in children with idiopathic headaches. Neurol Neurochir Pol 39(4 Suppl 1):S9-S16

55. Fujita M, Fujiwara J, Maki T, Shigeta M, Shibasaki K, Takahashi N, Takahashi M (2007) The efficacy of sodium valproate and a MRA finding in confusional migraine. Brain Dev 29(3):178-181

56. Avraham SB, Har-Gil M, Watemberg N (2010) Acute confusional migraine in an adolescent: response to intravenous valproate. Pediatrics 125(4):e956-e959

57. Siniatchkin M, Groppa S, Jerosch B et al (2007) Spreading photoparoxysmal EEG response is associated with an abnormal cortical excitability pattern. Brain 130(Pt 1):78-87

58. Bjørk MH, Stovner LJ, Nilsen BM, Stjern M, Hagen K, Sand T (2009) The occipital alpha rhythm related to the "migraine cycle" and headache burden: a blinded, controlled longitudinal study. Clin Neurophysiol 120(3):464-471

59. Sannita WG, Narici L, Picozza P (2006) Positive visual phenomena in space: a scientific case and a safety issue in space travel. Vision Res 46(14):2159-2165

60. Beckmann YY, Seçil Y, Kendir AI, Başoğlu M (2009) Chronic migraine: a prospective descriptive clinical study in a headache center population. Pain Pract 9(5):380-384

61. Syvertsen M, Helde G, Stovner LJ, Brodtkorb E (2007) Headaches add to the burden of epilepsy. J Headache Pain 8(4):224-230

62. Noseda R, Kainz V, Jakubowski M, Gooley JJ, Saper CB, Digre K, Burstein R (2010) A neural mechanism for exacerbation of headache by light. Nat Neurosci 13(2):239-245

63. Haglund MM, Schwartzkroin PA (1990) Role of Na-K pump potassium regulation and IPSPs in seizures and spreading depression in immature rabbit hippocampal slices. J Neurophysiol 63:225-239

64. Deprez L, Peeters K, Van Paesschen W et al (2007) Familial occipitotemporal lobe epilepsy and migraine with visual aura. Linkage to chromosome 9q. Neurology 68:1995-2002

65. Castro MJ, Stam AH, Lemos C et al (2009) First mutation in the voltage-gated Nav1.1 subunit gene SCN1A with co-occurring 
familial hemiplegic migraine and epilepsy. Cephalalgia 29(3):308-313

66. Vanmolkot KR, Kors EE, Hottenga JJ et al (2003) Novel mutations in the $\mathrm{Na}^{+}, \mathrm{K}^{+}$-ATPase pump gene ATP1A2 associated with familial hemiplegic migraine and benign familial infantile convulsions. Ann Neurol 54:360-366

67. Goadsby PJ, Charbit AR, Andreou AP, Akerman S, Holland PR (2009) Neurobiology of migraine. Neuroscience 161:327-341

68. Parisi P, Piccioli M, Villa MP, Buttinelli C, Kasteleijn-Nolst Trenate DGA (2008) Hypothesis on neurophysiopathological mechanisms linking epilepsy and headache. Med Hypotheses 70:1150-1154

69. Koutroumanidis M (2007) Panayiotopoulos syndrome: an important electroclinical example of benign childhood system epilepsy. Epilepsia 48:1044-1053

70. Bullmore E, Sporns O (2009) Complex brain networks: graph theoretical analysis of structural and functional systems. Nat Rev Neurosi 10(3):186-198

71. Moskowitz MA, Nozaki K, Kraig RP (1993) Neocortical spreading depression provokes the expression of C-fos protein- like immunoreactivity within trigeminal nucleus caudalis via trigeminovascular mechanisms. J Neurosci 13(3):1167-1177

72. Ayata C, Jin H, Kudo C, Dalkara T, Moskowitz MA (2006) Suppression of cortical spreading depression in migraine prophylaxis. Ann Neurol 59(4):652-661

73. Berger M, Speckmann EJ, Pape HC, Gorji A (2008) Spreading depression enhances human neocortical excitability in vitro. Cephalalgia 28:558-562

74. Calabresi P, Galletti F, Rossi C, Sarchielli P, Cupini LM (2007) Antiepileptic drugs in migraine: from clinical aspects to cellular mechanisms. Trends Pharmacol Sci 28(4):188-195

75. de Tommaso M, Guido M, Sardaro C et al (2008) Effects of topiramate and levetiracetam vs placebo on habituation of contingent negative variation in migraine patients. Neurosci Lett 442:81-85

76. Kasteleijn-Nolst Trenité DGA, Cantonetti L, Parisi P (2010) Visual stimuli, photosensitivity and photosensitive epilepsy. Chapter 94. In: Shorvon S, Guerrini R, Andermann F (eds) Common and uncommon causes of epilepsy. Cambridge University Press, Cambridge (in press) 